\title{
Bacterial battalions join war against cancer
}

Researchers battling cancer are finding an unexpected and powerful ally: bacteria. The idea is far from new-it was tried and abandoned years ago- - but researchers are now resurrecting it with a modern twist.

Scientists have known for more than 100 years that some bacteria can devour cancer. For example, animal studies have shown that an attenuated form of Salmonella - which prefers to replicate in cancer cells — can shrink tumors. The same is true of Clostridium novyi and Mycobacterium bovis BCG, now used to treat bladder cancer. But using live attenuated bacteria poses one big problem: toxicity. In some studies, nearly half of treated mice have died from toxic side effects.

Rather than use live bacteria, researchers are now genetically modifying bacterial toxins to fight cancer. Ira Pastan, chief of the laboratory of molecular biology at the US National Cancer Institute, has modified a powerful bacterial toxin called Pseudomonas exotoxin A. Pastan replaced the toxin's binding domain with antibodies specific for various cancers. Once the antibody binds to receptors on cancer cells, he says, the toxin enters and kills the cells.

One recombinant toxin is already on the market to treat persistent or recurrent cutaneous T-cell lymphoma. Many others are in phase 2 clinical trials to treat patients with colon, breast and ovarian cancers, mesothelioma, and CD25- and CD22-positive leukemias and lymphomas. Results from a phase 1 clinical trial showed that one toxin almost completely shrank tumors in 11 of 16 patients with hairy-cell leukemia.

Bacterial toxins will most likely be used in combination with other therapies, notes Stephen Leppla, a researcher at the US National Institute of Allergy and Infectious Diseases. "Most people imagine that cancers are going to be treated with combinations of treatments," Leppla says.

Because people develop antibodies to bacterial toxins, researchers may also need to develop a large variety of toxins, says Leppla. One solution, he says, may be to use antigenically distinct toxins in sequence so that antibodies to the first will not neutralize the second, and so forth.

Leppla has modified the anthrax toxin to become active only when cleaved by the enzyme urokinase, which is present on the surface of nearly all cancer cells. After one treatment in mice, the toxin reduced the tumor size of fibrosarcomas, melanoma and lung cancers by $65-92 \%$. After two treatments, it eliminated $88 \%$ of the fibrosarcomas and $17 \%$ of the melanomas. What's more, the toxin did not damage surrounding cells.

Another approach harnesses the affinity of some bacteria for cancer cells. A few years ago, Ananda Chakrabarty and his colleagues at the University of Illinois discovered that the bacterium Pseudomonas aeruginosa secretes a compound that kills cancer cells in culture. Chakrabarty isolated the molecule, azurin, long known to facilitate cellular redox reactions.

Further study revealed that azurin enters cells and binds to the normally unstable tumor suppressor protein p53 and stabilizes it. Inside

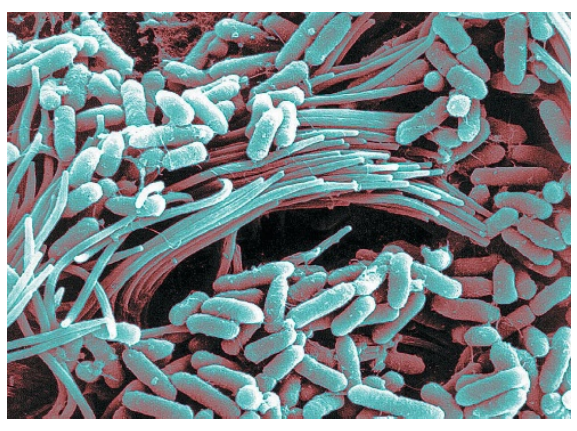

Blockbuster bugs: Bacteria, such as the rod-shaped Pseudomonas aeruginosa, could treat cancer.

cells, p53 acts like a gatekeeper to regulate a cell's repair and growth; mutations in p53 are commonly found in human cancers.

Last year, Chakrabarty and his colleagues showed that azurin shrank human melanoma tumors in immunodeficient mice by $60 \%$. None of the mice showed any signs of toxicity. "Purified bacterial proteins have been known to be involved in electron transfer, but not as agents of cancer cell death," Chakrabarty says. "This is an entirely new source of anticancer agents." Chakrabarty has already found other bacterial proteins that kill cancer.

To reduce the chances of a toxic reaction, Chakrabarty is trying to eliminate unnecessary pieces of the azurin protein. Pastan has also been identifying and deleting unnecessary gene sequences in his toxins. "We have decreased the size of our toxins and increased the antitumor activity," he says.

Gunjan Sinha, Frankfurt

\section{Taiwanese scientists brace for second season of SARS}

One of the nations hit hardest by severe acute respiratory syndrome (SARS), Taiwan is strengthening its surveillance and research on the infection, which claimed 180 lives in the country early this year.

Taiwanese scientists are spending about US\$58.6 million on developing a SARS vaccine. According to the researchers, preliminary results from the genetic sequence suggest the Taiwanese strain is slightly different from those in other SARS-affected areas, such as Hong Kong and Toronto.

At least 16 different projects on vaccinesincluding peptide vaccines, recombinant protein vaccines, DNA and viral vector vaccines and killed vaccines-are now under way. "Hopefully, we can finish the feasibility test in the primate model of the different vaccine approaches by next June, and select the best candidate vaccine for clinical trials," says MiHua Tao of the Institute of Biomedical Sciences of Academia Sinica in Taipei.

Researchers are also testing SARS-specific antibodies, horse serum and patients' convalescent serum as potential therapies.

Other teams are focusing on ways to detect the virus. One group has unpublished results showing that antibody detection of purified recombinant proteins of the SARS virus, combined with existing reverse-transcriptase poly-

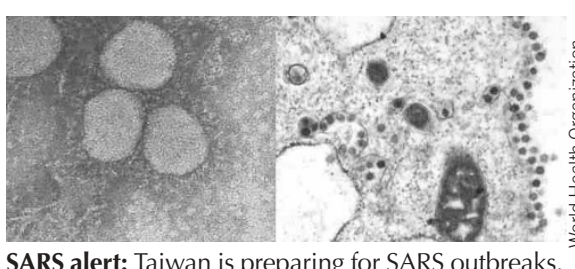

SARS alert: Taiwan is preparing for SARS outbreaks. merase chain reaction (RT-PCR), can enhance laboratory confirmation to $72.2 \%$ accuracy from $48.1 \%$ using RT-PCR alone.

Meanwhile, public health authorities in Taiwan are gearing up for potential SARS outbreaks. In September, Taiwan's Cabinet-level Department of Health announced that those who have fever will be quarantined at home for three days. The Department of Health has also established regulations pertaining to mass movement and rallies. Once probable cases are reported, any indoor gathering of more than 300 people will be prohibited.

Taiwan is also instituting mass vaccination of people above the age of 65 , and free flu vaccines will be provided to 300,000 high-risk professionals, including health-care and sanitary workers.

Yu-Tzu Chiu, Taipei 